S. Kawai

Nagoya Math. J.

Vol. 140 (1995), 151-166

\title{
SCALAR CURVATURES OF CONFORMAL METRICS ON $S^{n}$
}

\author{
SHIGEO KAWAI
}

\$0.

In this paper we consider the following problem: Given a smooth function $K$ on the $n$-dimensional unit sphere $S^{n}(n \geq 3)$ with its canonical metric $g_{0}$, is it possible to find a pointwise conformal metric $g=f g_{0}(f>0)$ which has $K$ as its scalar curvature? This problem was presented by J. L. Kazdan and F. W. Warner. The associated problem for Gaussian curvature in dimension 2 had been presented by L. Nirenberg several years before.

In both cases, the problems can be reduced to solving nonlinear partial differential equations: For $n=2$,

$$
-\Delta u+1=K \exp (2 u)
$$

where $f=\exp (2 u)$, and for $n \geq 3$,

$$
-\frac{4(n-1)}{n-2} \Delta u+n(n-1) u=K u^{\frac{n+2}{n-2}}, \quad u>0
$$

where $f=u^{\frac{4}{n-2}}$.

It is known that there exist functions $K$ with no solutions. This is shown by the obstruction of J. L. Kazdan and F. Warner which we now recall.

PROPOSITION 1 ([3], [13], [14]). If $u$ is a solution of the equation (1) (resp. (2)), then we have

$$
\begin{gathered}
\int_{S^{2}} \exp (2 u)\langle\nabla K, \nabla F\rangle d V_{g_{0}}=0 \\
\left(\text { resp. } \int_{S^{2}} u^{\frac{2 n}{n-2}}\langle\nabla K, \nabla F\rangle d V_{g_{0}}=0\right)
\end{gathered}
$$

for all spherical harmonics $F$ of degree 1 , where $d V_{g_{0}}$ denotes the canonical volume form on $S^{n}$.

Received September 20, 1994. 
Taking $K=c_{1}+c_{2} F_{0}$ with $c_{1}, c_{2}$ constants and $F_{0}$ a spherical harmonic of degree 1 , we conclude there is no solution of the equation (1) and (2). Note that the spherical harmonics of degree 1 are the restrictions of linear functions on $\mathbf{R}^{n+1}$ to the unit sphere and their critical sets have simple structures. Thus the nice sufficient conditions may include assumptions on the complexity of the critical set of $K$. Some existence resuits are known under symmetry assumptions on the function $K$ or in low dimensions ([2], [5], [6], [8], [10], [11], [12]). Recently Chang and Yang [7] presented a result in general dimensions.

The purpose of this paper is to present a consequence of min-max method, following Chen and Ding [8], applied to the $n$-dimensional case with $n \geq 3$. We also use the argument of Bahri and Coron [2] on the deformation of functions along the gradient line of a functional. For simplicity, we consider the following equation instead of the equation (2):

$$
\mathscr{L} u:=-\Delta u+\frac{n(n-2)}{4} u=K u^{\frac{n+2}{n-2}}, \quad u>0
$$

and assume that $\max K=1, n \geq 3$ throughout the paper.

THEOREM. Assume that a function $K$ on $S^{\mathrm{n}}$ with $\max K=1$ satisfies the following conditions:

(i) There exist nondegenerate local maximum points $a$ and $b$ such that

$$
\left(\frac{1}{2}\right)^{\frac{2}{n-2}}<\nu<K(b) \leq K(a)
$$

where

$$
\begin{aligned}
& \nu=\sup _{h \in \Gamma} \min _{x \in h([0,1])} K(x), \\
& \Gamma=\left\{h \in C^{0}\left([0,1], S^{\mathrm{n}}\right) \mid h(0)=a, h(1)=b\right\} .
\end{aligned}
$$

(ii) There exists an element $h_{0}$ in $\Gamma$ such that

$$
\min _{x \in h_{0}([0,1])} K(x)=\nu
$$

and $\Delta K(x)>0$ for any $x$ with $K(x)=\nu$.

(iii) There is no critical value of $K$ in the interval $(\nu, K(b))$.

Then we have a positive solution of the equation (3).

Though the restrictions on $K$ are rather stringent, we can apply the theorem, for example, to a function $K$ which has a saddle point $c$ with $K(c)>\left(\frac{1}{2}\right)^{\frac{2}{n-2}}$. 
To prove this theorem, we use the max-min method. Let us define functionals $F, G$ and $J$ on $W^{1,2}\left(S^{n}\right)$ as follows:

$$
\begin{aligned}
& F[u]=\oint|\nabla u|^{2}+\frac{n(n-2)}{4} \oint u^{2}, \\
& G[u]=\oint u_{+}{ }^{\frac{2 n}{n-2}}, \\
& J[u]=\oint K u_{+}{ }^{\frac{2 n}{n-2}}
\end{aligned}
$$

where $f_{f}=\left(\operatorname{vol} S^{n}\right)^{-1}\left(\int_{S^{n}} f d V_{g_{0}}\right)$ and $u_{+}(x)=\max \{u(x), 0\}$.

Taking families of functions $\phi_{a, \varepsilon}$ and $\phi_{b, \varepsilon}$ which will be specified later, we consider for some $\varepsilon>0$, the following max-min problem:

$$
\mu(\varepsilon)=\sup _{l \in L(\varepsilon)} \min _{u \in l([0,1])} J[u]
$$

where

$$
\begin{aligned}
H & =\left\{u \in W^{1,2}\left(S^{n}\right) \mid F[u]=1\right\}, \\
L(\varepsilon) & =\left\{l \in C^{0}([0,1], H) \mid l(0)=\Phi_{a, \varepsilon}, l(1)=\phi_{b, \varepsilon}\right\} .
\end{aligned}
$$

Under the assumptions of the theorem, this number $\mu(\varepsilon)$ turns out to be a critical value of $J_{\mid H}$ for sufficiently small $\varepsilon$, and we get a desired solution of the equation (3).

$\$ 1$.

In this section we make some preparation for the later parts. First we define several functions. For every point $p$ on $S^{n}, \sigma(p)$ denotes the streographic projection from $S^{n} \backslash\{p\}$ to $\mathbf{R}^{n}$, and $\rho(p)$ its inverse. Denoting the canonical metric of $\mathbf{R}^{n}$ and $S^{n}$ by $g$ and $g^{\prime}$ respectively, we have

$$
\rho(p)^{*} g^{\prime}=\left(\frac{2}{1+|x|^{2}}\right)^{2} g .
$$

Let us define $\delta(\alpha): \mathbf{R}^{n} \rightarrow \mathbf{R}^{n}$ by $\delta(\alpha)(x)=\frac{x}{\alpha}$. Then

$$
\delta(\alpha)^{*}\left(\rho(p)^{*} g^{\prime}\right)=\left(\frac{\alpha\left(1+|x|^{2}\right)}{\alpha^{2}+|x|^{2}}\right)^{2} \rho(p)^{*} g^{\prime},
$$


and consequently we obtain

$$
\operatorname{det} \delta(\alpha)=\left(\frac{\alpha\left(1+|x|^{2}\right)}{\alpha^{2}+|x|^{2}}\right)^{2}
$$

with respect to the spherical volume form $d V_{\rho(p) *^{\prime}}=\left(\frac{2}{1+|x|^{2}}\right)^{n} d V_{g}$ on $\mathbf{R}^{n}$. Let us define $\phi_{\alpha}=(\operatorname{det} \delta(\alpha))^{\frac{n-2}{2 n}}$ and $\phi_{p, \alpha}^{\prime}=\sigma(-p)^{*} \phi_{\alpha}$ where $-p$ denotes the antipodal point of $p$. Since

$$
\delta(\alpha)^{*}\left(\rho(-p)^{*} g^{\prime}\right)=\phi_{\alpha}^{\frac{4}{n-2}} \rho(-p)^{*} g^{\prime},
$$

the function $\phi_{p, \alpha}^{\prime}$ satisfies the equation

$$
-\frac{4(n-1)}{n-2} \Delta \phi_{p, \alpha}^{\prime}+n(n-1) \phi_{p, \alpha}^{\prime}=n(n-1) \phi_{p, \alpha}^{\frac{n+2}{n-2}}
$$

on $S^{n}$. In fact, every positive solution of this equation coincides with $\phi_{p, \alpha}^{\prime}$ for some $p$ and $\alpha$. Denoting the volume of $S^{n}$ by $\omega_{n}$, we have

$$
\begin{aligned}
\omega_{n} G\left[\phi_{p, \alpha}^{\prime}\right] & =\int_{\mathbf{R}^{n}} \phi_{\alpha}^{\frac{2 n}{n-2}} d V_{\rho(-p)^{*} g^{\prime}}=\int_{\mathbf{R}^{n}} u_{\alpha}^{\prime \frac{2 n}{n-2}} d V_{g}, \\
\omega_{n} J\left[\phi_{p, \alpha}^{\prime}\right] & =\int_{\mathbf{R}^{n}}\left(\rho(-p)^{*} K\right) \phi_{\alpha}^{\frac{2 n}{n-2}} d V_{\rho(-p)^{*} g^{\prime}} \\
& =\int_{\mathbf{R}^{n}}\left(\rho(-p)^{*} K\right) u_{\alpha}^{\prime \frac{2 n}{n-2}} d V_{g}, \\
\omega_{n} F\left[\phi_{p, \alpha}^{\prime}\right] & =\frac{n(n-2)}{4} \int_{S^{n}} \phi_{p, \alpha}^{\prime} \frac{2 n}{n-2} d V_{g^{\prime}} \\
& =\int_{\mathbf{R}^{n}}\left|\nabla u_{a}^{\prime}\right|^{2} d V_{g}
\end{aligned}
$$

where $u_{\alpha}^{\prime}=\left(\frac{2 \alpha}{\alpha^{2}+|x|^{2}}\right)^{\frac{n-2}{2}}$

Since $G\left[\phi_{p, \alpha}^{\prime}\right]=1$ and $F\left[\phi_{p, \alpha}^{\prime}\right]=\frac{n(n-2)}{4}$, we define $\phi_{p, \alpha}=\sqrt{\frac{4}{n(n-2)}} \phi_{p, \alpha}^{\prime}$ and $u_{\alpha}=\sqrt{\frac{4}{n(n-2)}} u_{\alpha}^{\prime}$. Then we have $\phi_{p, \alpha} \in H$ and

$$
\begin{gathered}
\mu_{0}:=\left(\frac{4}{n(n-2)}\right)^{\frac{n}{n-2}}=G\left[\phi_{p, \alpha}\right]=\max _{u \in H} G[u], \\
\omega_{n} J\left[\phi_{p, \alpha}\right]=\int_{\mathbf{R}^{n}}\left(\rho(-p)^{*} K\right) u_{\alpha}^{\frac{2 n}{n-2}} d V_{g} .
\end{gathered}
$$


The following property of functions $u_{\alpha}$ will be used: If we fix a ball $B(R)$ of radius $R$ centered at the origin,

$$
\begin{gathered}
\int_{B(R)^{c}} u_{\alpha}^{\frac{2 n}{n-2}} d V_{g}=O\left(\alpha^{n}\right), \int_{B(R)} u_{\alpha}^{\frac{2 n}{n-2}}|x|^{2} d V_{g} \sim \alpha^{2}, \\
\int_{B(R)} u_{\alpha}^{\frac{2 n}{n-2}}|x|^{3} d V_{g}= \begin{cases}O\left(\alpha^{3}\right) & (n \geq 4) \\
O\left(\alpha^{3} \log \left(\frac{1}{\alpha}\right)\right) & (n=3)\end{cases}
\end{gathered}
$$

as $\alpha \rightarrow 0$.

Next Proposition is a form of maximum principle by Stampacchia [17] (see also Kazdan and Warner [13]).

PROPOSITION 2. If a function $u \in W^{1,2}\left(S^{n}\right)$ satisfies the equation $\mathscr{L} u=f u_{+}^{\frac{n+2}{n-2}}$ for a function $f$, then $u \geq 0$.

Proof. Let us denote $u_{-}(x)=\min \{0, u(x)\}$. Then we have $u_{-} \in W^{1,2}\left(S^{n}\right)$ and

$$
\oint(\mathscr{L} u) u_{-}=\oint f u_{+}^{\frac{n+2}{n-2}} u_{-}=0
$$

Hence we obtain

$$
\oint\left(\left|\nabla u_{-}\right|^{2}+\frac{n(n-1)}{4} u_{-}^{2}\right)=0
$$

which implies $u_{-} \equiv 0$, i.e., $u \geq 0$.

We need the concept of renormalization or rescaling. Consider for $\alpha>0$ and $p \in S^{n}$, a diffeomorphism $\gamma_{\alpha, p}: S^{n} \rightarrow S^{n}$ defined by $\gamma_{\alpha, p}=\rho(-p) \circ \delta(\alpha) \circ \sigma(-p)$. For $u \in W^{1,2}\left(S^{n}\right), \alpha>0$ and $p \in S^{n}$, we define the rescaled function $\tilde{u}(\alpha, p)$ by

$$
\tilde{u}(\alpha, p)=\left(u \circ \gamma_{\alpha, p}\right)\left(\operatorname{det} \gamma_{\alpha, p}\right)^{\frac{n-2}{2 n}}=\left(u \circ \gamma_{\alpha, p}\right) \phi_{p, \alpha}^{\prime}
$$

In this notation, $\tilde{u}(\alpha, p)$ concentrates at $p$ as $\alpha \rightarrow 0$. The functionals $G$ and $F$ are invariant by rescaling, i.e.,

$$
G[\tilde{u}(\alpha, p)]=G[u], \quad F[\tilde{u}(\alpha, p)]=F[u] .
$$

Let us consider an inner product $\langle,\rangle_{1}$ on $W^{1,2}\left(S^{n}\right)$ defined by

$$
\langle u, v\rangle_{1}=\oint\langle\nabla u, \nabla v\rangle+\frac{n(n-2)}{4} \oint u v .
$$


This is apparently equivalent to the usual inner product in $W^{1,2}\left(S^{n}\right)$. The gradient of $J_{\mid H}$ with respect to $\langle,\rangle_{1}$, which we denote by $\operatorname{grad} J$, is given by

$$
(\operatorname{grad} J)[u]=\frac{2 n}{n-2}\left\{-J[u] u+\mathscr{L}^{-1}\left(K u_{+}^{\frac{n+2}{n-2}}\right)\right\}
$$

Proposition 3. If $\left\{u_{i}\right\}$ is a sequence in $H$ with $(\operatorname{grad} J)\left[u_{i}\right] \rightarrow 0$ and $\lim J\left[u_{i}\right]>\left(\frac{1}{2}\right)^{\frac{2}{n-2}} \mu_{0}$, then the equation (3) has a positive solution or a subsequence concentrates at exactly one point in $S^{n}$.

Proof. We follow the proof of Theorem 3.1 in Struwe [18] which proves the case $K \equiv 1$. For a function $f$, let us define a functional $E_{f}$ on $W^{1,2}\left(S^{n}\right)$ by

$$
E_{f}[u]=\frac{1}{2} F[u]-\frac{n-2}{2 n} \oint f u_{+}^{\frac{2 n}{n-2}}
$$

Then $(\operatorname{grad} J)\left[u_{i}\right] \rightarrow 0(i \rightarrow \infty)$ if and only if $\left\|E_{K}{ }^{\prime}\left[v_{i}\right]\right\| \rightarrow 0(i \rightarrow \infty)$ where $v_{i}=$ $J\left[u_{i}\right]^{\frac{2-n}{4}} u_{i}$. By the definition of $E_{K}$, we have

$$
-\Delta v_{i}+\frac{n(n-2)}{4} v_{i}-K v_{i_{+}}^{\frac{n+2}{n-2}} \rightarrow 0
$$

weakly as $i \rightarrow \infty$.

We note that the sequence $\left\{v_{i}\right\}$ is bounded in $W^{1,2}\left(S^{n}\right)$ because $F\left[u_{i}\right]=1$ and $\lim J\left[u_{i}\right]>\left(\frac{1}{2}\right)^{\frac{2}{n-2}} \mu_{0}$. Consequently a subsequence converges to $v \in$ $W^{1,2}\left(S^{n}\right)$ weakly. Then from the fact stated above, the function $v$ is a weak solution of the equation $\mathscr{L} v=K v_{+}^{\frac{n+2}{n-2}}$, and we have $v \geq 0$ by Proposition 2 . This weak solution $v$ is smooth from Theorem 3 in Trudinger [19]. Using maximum principle for smooth solution of elliptic equations, we obtain either $v \equiv 0$ or $v>0$ everywhere. In the latter case, we get a desired positive solution of the equation $\mathscr{L} v=K v^{\frac{n+2}{n-2}}$. Hence we may only consider the case that a subsequence of $\left\{v_{i}\right\}$, which is still denoted by $\left\{v_{i}\right\}$, converges to zero weakly.

Next we show $\left\|E_{K}{ }^{\prime}\left[v_{i}\right]\right\| \rightarrow 0$ and $\liminf E_{K}\left[v_{i}\right]<\frac{1}{n}\left(\frac{n(n-2)}{4}\right)^{\frac{n}{2}}$ implies that $\left\{v_{\imath}\right\}$ is relatively compact. Though this is true even if the weak limit does not equal to zero, we treat only the case $v_{i} \rightarrow 0$ weakly.

Since 


$$
o(1)=\left\langle v_{i}, E_{K}^{\prime}\left[v_{i}\right]\right\rangle=\oint\left|\nabla v_{i}\right|^{2}-\oint K v_{i+}^{\frac{2 n}{n-2}}+o(1),
$$

we obtain

$$
\mathcal{\nabla}\left|\nabla v_{i}\right|^{2}=n E_{K}\left[v_{i}\right]+o(1)<\left(\frac{n(n-2)}{4}\right)^{\frac{n}{2}}+o(1) .
$$

From the assumption $K \leq 1$ and Sobolev inequality, we get

$$
\begin{aligned}
o(1) & =\oint\left|\nabla v_{i}\right|^{2}-\oint K v_{i+}^{\frac{2 n}{n-2}}+o(1) \\
& \geq \oint\left|\nabla v_{i}\right|^{2}-\oint\left|v_{i}\right|^{\frac{2 n}{n-2}}+o(1) \\
& \geq \oint\left|\nabla v_{i}\right|^{2}-\left(\frac{4}{n(n-2)}\right)^{\frac{n}{n-2}}\left(\oint\left|\nabla v_{i}\right|^{2}\right)^{\frac{n}{n-2}}+o(1) \\
& \geq\left(\oint\left|\nabla v_{i}\right|^{2}\right)\left\{1-\left(\frac{4}{n(n-2)}\right)^{\frac{n}{n-2}}\left(\oint\left|\nabla v_{i}\right|^{2}\right)^{\frac{n}{n-2}}\right\}+o(1) .
\end{aligned}
$$

Thus it follows that $\left\|v_{i}\right\|_{1,2} \rightarrow 0$ as $i \rightarrow \infty$.

We can derive, as in the proof of Lemma 3.3 in [18], the following fact: There exist a sequence $\left\{x_{i}\right\}$ of points in $S^{n}$ with $x_{i} \rightarrow x_{0} \in S^{n}$, a sequence $\left\{\alpha_{i}\right\}$ of positive numbers with $\alpha_{i} \rightarrow 0$ and a nontrivial solution $v_{0}$ of the equation

$$
\mathscr{L} v_{0}=K\left(x_{0}\right) v_{0+}^{\frac{n+2}{n+2}}
$$

such that the sequence $\left\{w_{i}\right\}$ of functions defined by $w_{i}=v_{i}-\tilde{v}_{0}\left(\alpha_{i}, x_{i}\right)$ satisfies

$$
E_{K}\left[w_{i}\right]=E_{K}\left[v_{i}\right]-E_{K}\left[\tilde{v}_{0}\left(\alpha_{i}, x_{i}\right)\right]+o(1)=E_{K}\left[v_{i}\right]-E_{K\left(x_{0}\right)}\left[v_{0}\right]+o(1)
$$

and

$$
\left\|E_{K}{ }^{\prime}\left[w_{i}\right]\right\| \rightarrow 0 .
$$

The argument required to prove this is almost the same as in [18], and we only point out the differences.

First we use the following identity which can be proved by the method of Theorem 2 in [4]:

$$
\oint_{K} \cdot\left(\bar{v}_{i}\right)_{+}^{\frac{2 n}{n-2}}=\oint K \cdot\left(\bar{v}_{i}-v_{0}\right)_{+}^{\frac{2 n}{n-2}}+\oint K v_{0+}^{\frac{2 n}{n-2}}+o(1),
$$

where $\left\{\bar{v}_{i}\right\}$ is a rescaled sequence of $\left\{v_{i}\right\}$ and $v_{0}$ is the weak limit of $\left\{\bar{v}_{i}\right\}$. The corresponding relation for $K \equiv 1$ is used for example in p.173 (3.3) of [18] $\left(\bar{v}_{i}\right.$ and $v_{0}$ are written there as $\tilde{v}_{m}$ and $v^{0}$ respectively.). Secondly $v^{0}$ in [18] is a solution of 
the "limiting problem" (3.1) (p.169), while our "limiting problem" for $v_{0}$ becomes the equation (4). This is because rescalings are done from the points $x_{i}$, and we take the subsequence of $\left\{x_{i}\right\}$ so that it converges to a point $x_{0} \in S^{n}$.

We show in the following that $w_{i} \rightarrow 0$ in $W^{1,2}\left(S^{n}\right)$ which means that the sequence $\left\{v_{i}\right\}$ approaches $\tilde{v}_{0}\left(\alpha_{i}, x_{i}\right)$. From the equation (4), we get $v_{0}>0$ and $K\left(x_{0}\right)>$ 0 . Let us set $v_{0}^{\prime}=\left(\frac{4 K\left(x_{0}\right)}{n(n-2)}\right)^{\frac{n-2}{4}} v_{0}$. Then this positive function $v_{0}^{\prime}$ satisfies the equation

$$
-\frac{4(n-1)}{n-2} \Delta v_{0}^{\prime}+n(n-1) v_{0}^{\prime}=n(n-1) v_{0}^{\frac{n+2}{n-2}},
$$

and consequently $v_{0}^{\prime}=\phi_{p, \alpha}^{\prime}$ for some $p$ and $\alpha$. Hence we have

$$
F\left[v_{0}\right]=\left(\frac{n(n-2)}{4}\right)^{\frac{n}{2}} K\left(x_{0}\right)^{\frac{2-n}{2}}, \quad G\left[v_{0}\right]=\left(\frac{n(n-2)}{4 K\left(x_{0}\right)}\right)^{\frac{n}{2}}
$$

which implies

$$
E_{K}\left[w_{i}\right]=\frac{1}{n} J\left[u_{i}\right]^{\frac{2-n}{2}}-\frac{1}{n}\left(\frac{n(n-2)}{4}\right)^{\frac{n}{2}} K\left(x_{0}\right)^{\frac{2-n}{2}}+o(1)
$$

Suppose that $\left\{u_{i}\right\}$ satisfies the condition

$$
J\left[u_{i}\right]>\left(1+K\left(x_{0}\right)^{\frac{2-n}{2}}\right)^{-\frac{2}{n-2}} \mu_{0}
$$

where $\mu_{0}=\left(\frac{4}{n(n-2)}\right)^{\frac{n}{n-2}}$, then the inequality

$$
E_{K}\left[w_{i}\right]<\frac{1}{n}\left(\frac{n(n-2)}{4}\right)^{\frac{n}{2}}
$$

holds and we get $w_{i} \rightarrow 0$ in $W^{1,2}\left(S^{n}\right)$ because $\left\|E_{K}{ }^{\prime}\left[w_{i}\right]\right\| \rightarrow 0$. Since the function $\left(1+\frac{2-n}{t^{2}}\right)^{-\frac{2}{n-2}}$ of $t$ on the interval $(0,1]$ takes the maximum value $\left(\frac{1}{2}\right)^{\frac{2}{n-2}}$ at $t=$ 1 , the proof is completed.

The following is a variant of Mountain Pass Lemma.

Proposition 4. Let $f$ be a $C^{1}$-function defined on a closed smooth submanifold $X$ of a Hilbert space. Assume that for two points $p$ and $q$ in $X$,

$$
\mu=\sup _{c \in \Gamma} \min _{t \in[0,1]} f(c(t))<\min \{f(p), f(q)\}
$$


where

$$
\Gamma=\left\{c \in C^{0}([0,1], X) \mid c(0)=p, c(1)=q\right\} .
$$

Then for every sequence $\left\{c_{n}\right\}$ in $\Gamma$ with $\min _{t \in[0,1]} f\left(c_{n}(t)\right) \rightarrow \mu(n \rightarrow \infty)$, there exist sequences $\left\{c_{n}{ }^{\prime}\right\}$ in $\Gamma$, which can be taken as close to $\left\{c_{n}\right\}$ as possible, and $\left\{t_{n}\right\}$ in $[0,1]$ such that

$$
f\left(c_{n}{ }^{\prime}\left(t_{n}\right)\right)=\min _{t \in[0,1]} f\left(c_{n}^{\prime}(t)\right)
$$

and

$$
\left\|(\operatorname{grad} f)\left(c_{n}^{\prime}\left(t_{n}\right)\right)\right\| \rightarrow 0(n \rightarrow \infty) .
$$

If $X$ itself is a linear space, this proposition is proved in Aubin \& Ekeland [1] and Shuzhong [16]. The key ingredient in both proofs is Ekeland's variational principle ([9]) which is valid for functions on complete metric spaces. In our case, we consider the function $I$ defined by $I(c)=\max _{t \in[0,1]} f(c(t))$ on the space $\Gamma$, and apply Ekeland's variational principle to this function $I$. Since the space $\Gamma$ is a complete metric space, we can easily modify the proof to fit in with our case.

$\$ 2$.

The purpose of this section is to show that if $J_{\mid H}$ has no critical point, then there exists some constant $c>0$ such that

$$
\mu_{0} \nu<\mu(\varepsilon)<(K(b)-c) \mu_{0}
$$

for sufficiently small $\varepsilon$. For every $u \in W^{1,2}\left(S^{n}\right)$ with $u_{+} \not \equiv 0$, we define $P(u)=$ $\left(P(u)_{1}, P(u)_{2}, \cdots, P(u)_{n+1}\right)$ by the equality

$$
P(u)_{i}=\left(f u_{+}^{\frac{2 n}{n-2}} x_{i}\right)\left(f u_{+}^{\frac{2 n}{n-2}}\right)^{-1}
$$

where $\left(x_{1}, x_{2}, \cdots, x_{n+1}\right)$ is an orthogonal coordinate system of $\mathbf{R}^{n+1}$. When $|P(u)| \neq 0$, we write $Q(u)=P(u) /|P(u)|$ and $d(u)=|Q(u)-P(u)|$. If we are considering a sequence $\left\{u_{i}\right\}$ of functions which concentrates around at most one point, then $\left\{u_{i}\right\}$ actually concentrates if and only if $d\left(u_{i}\right) \rightarrow 0(i \rightarrow \infty)$. The following lemma can be proved by the same way as the proof of Lemma 1.1 in [8]. We present the proof because we need later some of the estimates in it.

Lemma 1. There exists a constant $C_{0}$ which depends only on the $C^{1}$-norm of $K$ such that for $u \in W^{1,2}\left(S^{n}\right)$ with $P(u) \neq 0$, 


$$
\left|\oint\{K-K(Q(u))\} u_{+}^{\frac{2 n}{n-2}}\right| \leq C_{0}(d(u))^{\frac{1}{3}} \mathcal{\int} u_{+}^{\frac{2 n}{n-2}}
$$

Proof. Let us define $B(r, Q)=\left\{x \in S^{n} \mid \operatorname{dist}(x, Q)<r\right\}$ where dist denotes the geodesic distance. Then we have

$$
\int_{S^{n} \backslash B(r, Q)}\left\{1-\sum_{i} x_{i} Q_{i}\right\} u_{+}^{\frac{2 n}{n-2}} d V^{\prime} \leq d(u) \int_{S^{n}} u_{+}^{\frac{2 n}{n-2}} d V^{\prime}
$$

and

$$
1-\sum_{i} x_{i} Q_{i} \geq \frac{r^{2}}{4}
$$

for every $x \in S^{n} \backslash B(r, Q)$. Taking $r=(d(u))^{\frac{1}{3}}$, we get

$$
\int_{S^{n} \backslash B(r, Q)} u_{+}^{\frac{2 n}{n-2}} d V^{\prime} \leq 4(d(u))^{\frac{1}{3}} \int_{S^{n}} u_{+}^{\frac{2 n}{n-2}} d V^{\prime} .
$$

Consequently we obtain

$$
\begin{aligned}
\left|\int_{S^{n}}\{K-K(Q(u))\} u_{+}^{\frac{2 n}{n-2}} d V^{\prime}\right| \leq & \left|\int_{B(r, Q)} \cdots\right|+\left|\int_{S^{n} \backslash B(r, Q)} \cdots\right| \\
\leq & (\max |\nabla K|) r \int_{S^{n}} u_{+}^{\frac{2 n}{n-2}} d V^{\prime} \\
& \quad+8(\max |K|)(d(u))^{\frac{1}{3}} \int_{S^{n}} u_{+}^{\frac{2 n}{n-2}} d V^{\prime} \\
\leq & C_{0}(d(u))^{\frac{1}{3}} \int_{S^{n}} u_{+}^{\frac{2 n}{n-2}} d V^{\prime}
\end{aligned}
$$

which is the desired result.

Lemma 2. Under the assumption on $K$ in the theorem, we have $\mu(\varepsilon)>\mu_{0} \nu$ for sufficiently small $\varepsilon$.

Proof. Using the path $h_{0}$ in the assumption (ii) of the theorem. We set $l_{\varepsilon}(t)=$ $\phi_{h_{0}(t), \varepsilon}$ for $t \in[0,1]$. To prove $\mu(\varepsilon)>\mu_{0} \nu$, we have only to show

$$
\int K\left(\phi_{h_{0}(t), \varepsilon}\right)^{\frac{2 n}{n-2}}>\mu_{0} \nu
$$

for $0 \leq t \leq 1$. Let us set $N_{\delta}=\left\{x \in h_{0}([0,1]) \mid \operatorname{dist}(x, A)<\delta\right\}$ where $A=$ $\left\{y \in h_{0}([0,1]) \mid K(y)=\nu\right\}$. Then we can choose a small $\delta$ so that $\Delta K_{\mid N_{\delta}}>0$. 
We first show the inequality (5) for $t$ with $h_{0}(t) \in S^{n} \backslash N_{\delta}$. Take a sufficiently small $\beta>0$ such that

$$
K(\zeta) \geq \nu+\beta
$$

for every $\zeta \in h_{0}([0,1]) \backslash N_{\delta}$, and let $\varepsilon$ be sufficiently small so that

$$
d\left(\phi_{\zeta, \varepsilon}\right) \leq\left(\frac{\beta}{2 C_{0}}\right)^{3} .
$$

Here note that $d\left(\phi_{\zeta, \varepsilon}\right)$ does not depend on $\zeta$. Then by Lemma 1 , we have

$$
\begin{aligned}
\int K\left(\phi_{h_{0}(t), \varepsilon}\right)^{\frac{2 n}{n-2}} & \geq\left\{K\left(h_{0}(t)\right)-C_{0}\left(d\left(\phi_{h_{0}(t), \varepsilon}\right)^{\frac{1}{3}}\right\} \mathcal{S}\left(\phi_{h_{0}(t), \varepsilon}\right)^{\frac{2 n}{n-2}}\right. \\
& \geq\left(\nu+\beta-\frac{\beta}{2}\right) \mathcal{\delta}\left(\phi_{h_{0}(t), \varepsilon}\right)^{\frac{2 n}{n-2}} \\
& \geq\left(\nu+\frac{\beta}{2}\right) \mu_{0} \\
& >\mu_{0} \nu .
\end{aligned}
$$

Next we consider the case $h_{0}(t) \in N_{\delta}$. Due to the continuity of $J$ and the relative compactness of $N_{\delta}$, it suffices to show that for each $\zeta \in N_{\delta}$, there is a num ber $\varepsilon(\zeta)$ such that the inequality (5) holds for $\varepsilon$ with $\varepsilon \leq \varepsilon(\zeta)$.

Since $\rho(-\zeta)$ is a conformal diffeomorphism,

$$
\Delta\left(\rho(-\zeta)^{*} K\right)=\Delta(K \circ \rho(-\zeta))=(d K)(\tau(\rho(-\zeta)))+f(\Delta K) \circ(\rho(-\zeta))
$$

where $f$ is a positive $C^{\infty}$ function on $\mathbf{R}^{n}$ and $\tau(\rho(-\zeta))$ denotes the tension field of the map $\rho(-\zeta)$. By straight forward computation, we see $\tau(\rho(-\zeta))(0)=0$ which implies

$$
\Delta\left(\rho(-\zeta)^{*} K\right)(O)=f(O)(\Delta K)(\zeta) .
$$

Denoting by $B(R)$ the ball of radius $R$ centered at the origin $O$ and considering Taylor expansion of $\rho(-\zeta)^{*} K$ at the origin, we get

$$
\begin{aligned}
\omega_{n} J\left[\phi_{\zeta, \varepsilon}\right]= & \int_{B(R)}\left(\rho(-\zeta)^{*} K\right) u_{\varepsilon}^{\frac{2 n}{n-2}} d V_{g}+\int_{B(R)^{c}}\left(\rho(-\zeta)^{*} K\right) u_{\varepsilon}^{\frac{2 n}{n-2}} d V_{g} \\
= & \int_{B(R)}\left\{\left(\rho(-\zeta)^{*} K\right)(O)+\sum_{i}\left(\frac{\partial}{\partial x_{i}}\left(\rho(-\zeta)^{*} K\right)(O)\right) x_{i}\right. \\
& \left.+\sum_{i, j}\left\{\frac{\partial^{2}}{\partial x_{i} \partial x_{j}}\left(\rho(-\zeta)^{*} K\right)(O)\right) x_{i} x_{j}+O\left(|x|^{3}\right)\right\} u_{\varepsilon}^{\frac{2 n}{n-2}} d V_{g} \\
& +\int_{B(R)^{c}}\left(\rho(-\zeta)^{*} K\right) u_{\varepsilon}^{\frac{2 n}{n-2}} d V_{g}
\end{aligned}
$$




$$
\begin{aligned}
= & K(\zeta) \mu_{0} \omega_{n}+\frac{f(O)}{n}(\Delta K)(\zeta) \int_{B(R)} u_{\varepsilon}^{\frac{2 n}{n-2}}|x|^{2} d V_{g} \\
& +\int_{B(R)} O\left(|x|^{3}\right) u_{\varepsilon}^{\frac{2 n}{n-2}} d V_{g}+O\left(\varepsilon^{n}\right) .
\end{aligned}
$$

In this calculation, we used the property that the functions $u_{\varepsilon}^{\frac{2 n}{n-2}}$ are radial and hence the integrals over $B(R)$ involving $x_{i} u_{\varepsilon}^{\frac{2 n}{n-2}}$ and $x_{i} x_{j} u_{\varepsilon}^{\frac{2 n}{n-2}}(i \neq j)$ must vanish.

Note that

$$
\int_{B(R)} u_{\varepsilon}^{\frac{2 n}{n-2}}|x|^{2} d V_{g} \sim \varepsilon^{2}, \int_{B(R)} u_{\varepsilon}^{\frac{2 n}{n-2}}|x|^{3} d V_{g}=O\left(\varepsilon^{3}\right) \text { or }=O\left(\varepsilon^{3} \log \left(\frac{1}{\varepsilon}\right)\right) .
$$

Since $K(\zeta)>\nu, f(O)(\Delta K)(\zeta)>0$, we obtain a number $\varepsilon(\zeta)$ such that the inequality (4) holds for every $\varepsilon$ with $\varepsilon \leq \varepsilon(\zeta)$. This completes the proof of the lemma.

PROPOSITION 5. If the function $K$ satisfies the assumption in the theorem, then either we have a critical point of the functional J, or there exists a positive constant $c$ such that

$$
\mu(\varepsilon)<(K(b)-c) \mu_{0}
$$

for every sufficiently small $\varepsilon$.

Proof. By the definition, the functions $\phi_{a, \varepsilon}$ and $\phi_{b, \varepsilon}$ concentrate around the points $a$ and $b$ respectively for small $\varepsilon$. To investigate the behavior of $\Phi_{s} \phi_{a, \varepsilon}$ and $\Phi_{s} \phi_{b, \varepsilon}$ by the flow $\left\{\Phi_{s}\right\}(s>0)$ on $H$ generated by $\operatorname{grad} J$, we use the argument in [2]. In that paper, the set of functions which concentrate at $p$ points is denoted by $W(p, \varepsilon)$ and the definition of "center" $a_{i}$ is different from that of $Q$. However if a function sufficiently concentrates at exactly one point, we may think that our $Q$ is nearly equal to $a_{1}$ in [2], and $\varepsilon \rightarrow 0$ in our notation corresponds to $\lambda_{1} \rightarrow \infty$ in [2].

The equations (121) and (122) in [2] imply that if a function sufficiently concentrates around a nondegenerate local maximum point, then it more and more concentrates around this point by the flow $\left\{\Phi_{s}\right\}$ as $s \rightarrow \infty$ (Incidentally "- " in the right hand side of the equation (122) should be replaced by “+”.). Though [2] treats mainly 3 -dimensonal case, this property is valid in all dimensions if we consider functions which concentrate at exactly one point. Thus if $\varepsilon$ is sufficiently small, the functions $\Phi_{s} \phi_{a, \varepsilon}$ and $\Phi_{s} \phi_{b, \varepsilon}$ concetrate more and more around points $a$ and $b$ respectively, and points $Q\left(\Phi_{s} \phi_{a, \varepsilon}\right), Q\left(\Phi_{s} \phi_{b, \varepsilon}\right)$ are well defined for all $s \geq 0$.

By the definition of $\mu(\varepsilon)$, there exists $l \in L(\varepsilon)$ such that $\min J_{\mid l([0,1])}$ is close 
to $\mu(\varepsilon)$. Then we have for every $v \in l([0,1])$,

$$
J\left[\Phi_{s}(v)\right] \geq\left(\frac{1}{2}\right)^{\frac{2}{n-2}} \mu_{0}, \quad\left(\operatorname{grad} J\left[\Phi_{s}(v)\right] \rightarrow 0(s \rightarrow+\infty) .\right.
$$

It follows from Section 1, Proposition 3 that there exists a critical point of the functional $J$ or a subsequence $\left\{\Phi_{s_{k}}(v)\right\}$ concentrates at one point in $S^{n}$. If the first case does not occur, we have not only $d\left(\Phi_{s_{k}}(v)\right) \rightarrow 0$ but also $d\left(\Phi_{s}(v)\right) \rightarrow 0$ as $s \rightarrow+\infty$. Consequently $Q\left(\Phi_{s}(v)\right)$ is well defined for every sufficiently large $s$.

From the compactness of $l([0,1])$, we get a sufficiently large constant $s_{0}$ such that $Q\left(\Phi_{s}(v)\right)$ is well defined for all $v \in l([0,1])$ and all $s \geq s_{0}$. Thus considering the path made by $\left\{\Phi_{s}\left(\phi_{a, \varepsilon}\right)\right\}_{0 \leq s \leq 2 s_{0}},\left\{\Phi_{2 s_{0}}(l([0,1]))\right\}$ and $\left\{\Phi_{s}\left(\phi_{b, \varepsilon}\right)\right\}_{0 \leq s \leq 2 s_{0}}$, we obtain a path $l^{\prime}$ between $\phi_{a, \varepsilon}$ and $\phi_{b, \varepsilon}$ such that $Q(v)$ is well defined and $d(v)$ is sufficiently small for every element $v$ in $l^{\prime}$.

Since $\left\{Q(v) \mid v \in l^{\prime}([0,1])\right\}$ is a continuous path between $a$ and $b$, there exists an element $v_{0} \in l^{\prime}([0,1])$ such that $K\left(Q\left(v_{0}\right)\right)<K(b)$ by the assumption (i). Because $v_{0}$ sufficiently concentrates at $Q\left(v_{0}\right)$, we have

$$
J\left[v_{0}\right]<\left(K(b)-c^{\prime}\right) \mu_{0}
$$

for some positive constant $c^{\prime}$. Thus the inequality $J_{\mid l([0,1])} \leq \min J_{\mid l^{\prime}([0,1])}$ and the closeness of $\min J_{\mid l([0,1])}$ to $\mu(\varepsilon)$ complete the proof.

Let us fix a small $\varepsilon_{0}$ so that Proposition 5 and Lemma 2 hold for every $\varepsilon$ $\leq \varepsilon_{0}$, and let us write $\mu=\mu\left(\varepsilon_{0}\right)$ and $L=L\left(\varepsilon_{0}\right)$ for simplicity.

PROPOSITION 6. There exist positive constants $\alpha_{0}, \delta_{0}$ with the following property: If a sequence $\left\{v_{k}\right\}$ in $H$ satisfies

$$
J\left[v_{k}\right]>\mu-\delta_{0}, \quad P\left(v_{k}\right) \rightarrow \zeta \in S^{n}
$$

as $k \rightarrow \infty$, then

$$
K(\zeta) \geq \nu+\alpha_{0}
$$

Proof. From Lemma 2, we get positive constants $\alpha_{0}^{\prime}$ and $\delta_{0}$ such that

$$
\mu-\delta_{0} \geq \mu_{0}\left(\nu+\alpha_{0}^{\prime}\right) .
$$

Then from Lemma 1, the following inequalities hold:

$$
\left(\nu+\alpha_{0}^{\prime}\right) \mu_{0} \leq \mu-\delta_{0}<J\left[v_{k}\right] \leq\left\{K\left(Q\left(v_{k}\right)\right)+C_{0}\left(d\left(v_{k}\right)\right)^{\frac{1}{3}}\right\} \mu_{0} .
$$


Since $d\left(v_{k}\right) \rightarrow 0$ and $K\left(Q\left(v_{k}\right)\right) \rightarrow K(\zeta)$ as $k \rightarrow 0$, we obtain the desired result.

§3.

In this section we complete the proof of the theorem. Since we have only to consider the second case in Proposition 5, let us choose $\varepsilon_{1}$ so small that $\mu$ $<\mu_{0}\left(K(b)-\varepsilon_{1}\right)$ and take the set $U_{d}=\{u \in H \mid P(u) \neq 0, K(Q(u)) \leq K(b)-$ $\left.\varepsilon_{1}, d(u) \leq d\right\}$. From now on, $J^{-1}(a, b)$ (resp. $J^{-1}[a, b]$ ) denotes the subset $\{u \in$ $H \mid a<J[u]<b\}$ (resp. $\{u \in H \mid a \leq J[u] \leq b\}$ ).

The proof of the following lemma is almost the same as that of Lemma 5.1 in [8] and we omit it.

Lemma 3. There exist positive constants $\delta, d$ with $K(b) \mu_{0}-\delta>\mu+\delta$, and $a$ continuous map $\mathscr{T}: H \rightarrow H$ such that

(1) $J[\mathscr{T} u] \geq J[u]$ for every $u \in H$,

(2) $\mathscr{T}\left(J^{-1}\left(\mu-\delta, K(b) \mu_{0}\right) \cap U_{d}\right) \subset J^{-1}\left(\mu+\delta, K(b) \mu_{0}\right)$,

(3) $\mathscr{T}(u)=u$ for every $u \in J^{-1}\left(K(b) \mu_{0}-\delta, \mu_{0}\right)$,

(4) $\mathscr{T}\left(H \backslash U_{d}\right) \subset H \backslash U_{d}$.

Proof of the theorem. Choose a sequence $\left\{l_{k}\right\}_{k=1,2, \ldots}$ in $L$ such that

$$
\min _{u \in l_{k}[0,1]} J[u]>\mu-\delta, \min _{u \in l_{k}[0,1]} J[u] \rightarrow \mu
$$

as $k \rightarrow \infty$. By (1) and (3) in Lemma 3, we have $\mathscr{T}\left(l_{k}\right) \in L$ and

$$
\min _{\mathscr{T}\left(l_{k}(0,1)\right)} J[u] \rightarrow \mu .
$$

Also we get from (2) and (4) in Lemma 3,

$$
\left(\mathscr{T}\left(l_{k}[0,1]\right)\right) \cap U_{d} \subset J^{-1}\left(\mu+\delta, K(b) \mu_{0}\right) .
$$

By virtue of Proposition 4, we obtain $m_{k} \in L$ and $v_{k} \in m_{k}[0,1]$ such that

$$
J\left[v_{k}\right]=\min _{u \in m_{k}[0,1]} J[u], \quad J\left[v_{k}\right] \rightarrow \mu, \quad(\operatorname{grad} J)\left[v_{k}\right] \rightarrow 0 .
$$

Moreover we can take $m_{k}$ as close to $\mathscr{T}\left(l_{k}\right)$ as possible. Hence the relation (6) implies $v_{k} \in H \backslash U_{d}$ for sufficiently large $k$.

Since $(\operatorname{grad} J)\left[v_{k}\right] \rightarrow 0$, and $J\left[v_{k}\right] \rightarrow \mu,\left\{v_{k}\right\}$ concentrates at most one point from Proposition 3 and Lemma 2. Because $v_{k} \in H \backslash U_{d}$, we have only two cases:

(a) A subsequence of $\left\{v_{k}\right\}$ converges in $W^{1,2}$-norm.

(b) $P\left(v_{k}\right) \rightarrow \zeta \in S^{n}$ with $K(\zeta)>K(b)-\varepsilon_{1}$. 
In the case (b), $J\left[v_{k}\right] \rightarrow \mu_{0} K(\zeta)>\mu_{0}\left(K(b)-\varepsilon_{1}\right)$ which contradicts the fact $J\left[v_{k}\right] \rightarrow \mu<\mu_{0}\left(K(b)-\varepsilon_{1}\right)$. Thus only the case (a) occurs and we get a critical function $v$ in $H$ for functional $J$. Namely $v$ weakly satisfies the equation

$$
-\Delta v+\frac{n(n-2)}{4} v=\lambda K v_{+}^{\frac{2 n}{n-2}}
$$

for some constant $\lambda$. Hence from Proposition 2, a constant multiple of $v$ gives the desired solution of the equation (3). Thus the proof of the theorem is completed.

\section{REFERENCES}

[1] Aubin, J. P. and Ekeland, I., Applied Nonlinear Analysis, Wiley-Interscience, New York, 1984.

[2] Bahri, A. and Coron, C., The scalar curvature problem on the three-dimensional sphere, J. Funct. Anal., 95 (1991), 106-172.

[ 3 ] Bourguignon, J. P. and Ezin, J. P., Scalar curvature functions in a conformal class of metrics and conformal transformations, Trans. Amer. Math. Soc., 301 (1987), $723-736$.

[4] Brezis, H. and Lieb, E., A relation between pointwise convergence of functions and convergence of functionals, Proc. Amer. Math., Soc., (1983), 486-490.

[ 5 ] Chang, S.-Y. and Yang, P. C., Prescribing Gaussian curvature on $S^{2}$, Acta Math., 159 (1987), 215-259.

[6] Chang, S.-Y. and Yang, P. C., Conformal deformation of metrics on $S^{2}$, J. Diff. Geom., 27 (1988), 259-296.

[ 7 ] Chang, S.-Y. and Yang, P. C., A perturbation result in prescribing scalar curvature on $S^{n}$, Duke Math. J., 64 (1991), 27-69.

[8] Chen, W. and Ding, W., Scalar curvatures on $S^{2}$, Trans. Amer. Math. Soc., 303 (1987), 365-382.

[9] Ekeland, I., On the variational principle, J. Math. Anal. Appl., 47, 324-353.

[10] Escobar, J. F. and Schoen, R., Conformal metrics with prescribed scalar curvature, Invent. Math., 86 (1986), 243-254.

[11] Han, Z. -C., Prescribing Gaussian curvature on $S^{2}$, Duke Math. J., 61 (1990). $679-703$.

[12] Hong, C., A best constant and the Gaussian curvature, Proc. Amer. Math. Soc., 97 (1986), 737-747.

[13] Kazdan, J. L. and Warner, F. W., Curvature functions for compact 2-manifolds, Ann. of Math., 99 (1974), 14-74.

[14] Kazdan, J. L. and Warner, F. W., Scalar curvature and conformal deformation of Riemannian structure, J. Diff. Geom., 10 (1975), 113-134.

[15] Moser, J., On a nonlinear problem in differential geometry, Dynamical System, Academic Press, New York, 1973.

[16] Shuzhong, S., Ekeland's variational principle and the mountain pass lemma, Acta Mathematica Sinica, 1 (1985), 348-355.

[17] Stampacchia, G., On some regular multiple integral problems in the calculus of variations, Comm. Pure Appl. Math., 16 (1963), 383-421.

[18] Struwe, M., Variational Methods, Springer, Berlin Heidelberg, 1990. 
[19] Trudinger, N. S., Remarks concerning the conformal deformation of Riemannian structures on compact manifolds, Ann. Scuola Norm. Sup. Pisa, 22 (1968), $265-274$

Department of Mathematics

Faculty of Education

Saga University

Saga 840, Japan 